\title{
Low Endogenous G-Protein-Coupled Receptor Kinase 2 Sensitizes the Immature Brain to Hypoxia-Ischemia- Induced Gray and White Matter Damage
}

\author{
Cora H. A. Nijboer, ${ }^{1,2}$ Annemieke Kavelaars, ${ }^{1}$ Anne Vroon, ${ }^{1}$ Floris Groenendaal, ${ }^{2}$ Frank van Bel, ${ }^{2}$ and Cobi J. Heijnen ${ }^{1}$ \\ ${ }^{1}$ Laboratory of Psychoneuroimmunology and ${ }^{2}$ Department of Neonatology, University Medical Center Utrecht, 3584 EA Utrecht, The Netherlands
}

Hypoxic-ischemic brain injury is regulated in part by neurotransmitter and chemokine signaling via G-protein-coupled receptors (GPCRs). GPCR-kinase 2 (GRK2) protects these receptors against overstimulation by inducing desensitization. Neonatal hypoxic-ischemic brain damage is preceded by a reduction in cerebral GRK2 expression. We determined the functional importance of GRK2 in hypoxic-ischemic brain damage.

Nine-day-old wild-type and GRK2 ${ }^{+/-}$mice with a $\sim 50 \%$ reduction in GRK2 protein were exposed to unilateral carotid artery occlusion and hypoxia. In GRK2 ${ }^{+/-}$animals, gray and white matter damage was aggravated at 3 weeks after hypoxia-ischemia. In addition, cerebral neutrophil infiltration was increased in GRK2 ${ }^{+-}$animals. Neutrophil depletion reduced brain damage, but neuronal loss was still more pronounced in GRK2 ${ }^{+/-}$animals. Onset of neuronal loss was advanced in GRK2 ${ }^{+/-}$animals regardless of neutrophil depletion. White matter injury was advanced in GRK2 ${ }^{+/-}$animals and was not affected by neutrophil depletion. Activation/infiltration of microglia/macrophages was stronger in GRK2 ${ }^{+/-}$brains but only occurred $24 \mathrm{~h}$ after hypoxia-ischemia and is therefore not the primary cause of increased damage. During hypoxia, cerebral blood flow was reduced to the same extent in both genotypes. In vitro, GRK2 ${ }^{+/-}$ hippocampal slices and cerebellar granular neurons were more sensitive to glutamate-induced death.

We propose the novel concept that the kinase GRK2 regulates onset and magnitude of hypoxic-ischemic brain damage. Increased gray and white matter damage in GRK2 ${ }^{+/-}$animals was not dependent on infiltrating neutrophils and occurred before microglia/macrophage activation was detected. Collectively, our data suggest that cerebral GRK2 has an important endogenous neuroprotective role in ischemic cerebral damage.

Key words: GPCR; GRK2; hypoxia-ischemia; knock-out mice; neonatal; neuroinflammation

\section{Introduction}

Perinatal hypoxia-ischemia (HI) is a major cause of morbidity and mortality in the human newborn (Cowan et al., 2003; Ferriero, 2004). Impaired cerebral supply of nutrients and oxygen during $\mathrm{HI}$ followed by reperfusion induces a complex sequence of events that ultimately leads to brain damage. Glutamate excitotoxicity through overstimulation of both metabotropic glutamate receptors (mGluRs) and NMDA receptors initiates cerebral damage (Pellegrini-Giampietro, 2003; Johnston, 2005). Moreover, HI induces an inflammatory reaction characterized by increased numbers of cerebral neutrophils and microglial/macrophage activation. Prevention of cerebral neutrophil infiltration markedly reduced brain damage (Hudome et al., 1997; Palmer et

Received 0ct. 22, 2007; revised Jan. 30, 2008; accepted Feb. 8, 2008.

This work was supported by the Wilhelmina Children's Hospital Research Fund, the European Commission (Sixth Framework Program, contract \#LSHM-CT-2006-036534, neobrain), and in part by Zorgonderzoek Nederland/ Medische Wetenschappen Veni Grant 916.66 .026 (A.V.). The premixed gas used in this study was kindly provided by HoekLoos Medical (Eindhoven, The Netherlands). We thank S. van Neerven and J. Zijlstra for excellent technical assistance.

Correspondence should be addressed to Dr. Cobi J. Heijnen, University Medical Center Utrecht, Room KC 03.068.0, Lundlaan 6, 3584 EA Utrecht, The Netherlands. E-mail: C.Heijnen@umcutrecht.nl.

DOI:10.1523/JNEUROSCI.4769-07.2008

Copyright $\odot 2008$ Society for Neuroscience $\quad$ 0270-6474/08/283324-09\$15.00/0 al., 2004). Inhibition of microglial/macrophage activation can also attenuate brain damage (Clarkson et al., 2005).

Both neurotransmitters that initiate neuronal loss and chemokines that regulate cerebral infiltration/activation of inflammatory cells, signal through G-protein-coupled receptors (GPCRs). Agonist-induced desensitization and internalization of GPCRs comprise an important regulatory process to ensure adequate signaling and to prevent damage by overstimulation of GPCR. GPCR kinase (GRK)-dependent GPCR phosphorylation initiates this agonist-induced adaptive response that results in attenuation of signaling and removal of receptors from the cell surface (for review, see Pitcher et al., 1998a). GRK2 is the most widely studied member of the GRK family. It is known that the level of intracellular GRK2 controls chemokine-induced migration of inflammatory cells and signaling through receptors involved in activation of microglia/macrophages (Vroon et al., 2006). Moreover, changes in intracellular levels of GRK2 can affect inflammatory cell infiltration in vivo as well (Fan and Malik, 2003; Vroon et al., 2004, 2005). For example, we described advanced onset of experimental autoimmune encephalomyelitis in mice with a partial deletion of GRK2 (GRK2 ${ }^{+/}$animals) in association with increased early cerebral infiltration of inflammatory cells (Vroon et al., 2005). In addition to an important role in 
chemokine receptor regulation, GRK2 can modulate signaling via group I metabotropic glutamate receptors (mGluR1 and mGluR5). Overexpression of GRK2 attenuates mGluR1dependent cell death and signaling (Dhami et al., 2002; Sorensen and Conn, 2003). Moreover, GRK2 does not only regulate the responsiveness of cells via GPCR desensitization but can also interfere with cellular function by directly interacting with intracellular signaling molecules (Ribas et al., 2007). Together, GRK2 can function as a pivotal molecule in the regulation of many receptors and downstream signaling molecules involved in cerebral damage after a hypoxic-ischemic event.

The cellular level of GRK2 can be modulated by cytokines and reactive oxygen species, mediators that are upregulated after $\mathrm{HI}$ (Lombardi et al., 1999, 2002, 2007; Ramos-Ruiz et al., 2000; Arraes et al., 2006; Buonocore and Groenendaal, 2007; Cobelens et al., 2007). Interestingly, neonatal HI-induced brain damage is associated with a decrease in cerebral GRK2 protein expression before damage becomes apparent (Lombardi et al., 2004). These data suggest that decreased GRK2 may contribute to the pathophysiological process leading to $\mathrm{HI}$-induced brain damage.

Therefore, we determined the functional importance of GRK2 for $\mathrm{HI}$ brain damage. We compared $\mathrm{HI}$-induced cerebral damage in neonatal wild-type (WT) and GRK2 ${ }^{+/-}$animals, which express a $50 \%$ reduction in GRK2 levels and determined the contribution of neutrophils. Finally, we analyzed sensitivity of GRK2 ${ }^{+/-}$hippocampal slices and cerebellar granular neurons for glutamate-induced cell death in vitro.

\section{Materials and Methods}

Animals. Experiments were performed in accordance with international guidelines and were approved by the University Medical Center Utrecht experimental animal committee.

At postnatal day 9 , heterozygous GRK2 ${ }^{+} /^{-}$[homozygous GRK2 deletion is embryonically lethal (Jaber et al., 1996)] and WT C57BL/6 littermates were anesthetized ( $5 \mathrm{~min})$ with isoflurane $(3.0 \%$ induction, $1.0 \%$ maintenance) in $\mathrm{O}_{2}: \mathrm{N}_{2} \mathrm{O}(1: 1)$. The right common carotid artery was occluded by thermocauterization, xylocaine $(100 \mathrm{mg} / \mathrm{ml}$; AstraZeneca, Zoetermeer, The Netherlands) was applied, and wounds were closed. Throughout surgery, pups were kept on a heated mattress. After minimal $1 \mathrm{~h}$ recovery, pups were exposed to $10 \% \mathrm{O}_{2}$ in $\mathrm{N}_{2}$ for $45 \mathrm{~min}$ and returned to their dams. Sham-treated controls underwent anesthesia and incision only.

For depletion of neutrophils, $28 \mu \mathrm{l} / \mathrm{g}$ body weight rabbit anti-mouse polymorphonuclear (PMN) antibody or normal rabbit serum (Accurate Antibodies, Westbury, NY) prediluted 1:10 in sterile saline was injected intraperitoneally $20 \mathrm{~h}$ before and directly after HI. Depletion of circulating neutrophils was determined by a differential count.

Histological preparations. Mice received an overdose of pentobarbital and were perfused with $4 \%$ paraformaldehyde in PBS. Brains were embedded in paraffin. Coronal sections $(8 \mu \mathrm{m})$ cut at approximately -1.8 $\mathrm{mm}$ (at hippocampal level) and $+0.7 \mathrm{~mm}$ (at striatal level) from the bregma were stained with hematoxylin-eosin (HE) (Klinipath, Duiven, The Netherlands) and scored in a blinded way on a scale from $0-3$ in six regions of the parietal cortex and in five regions of the hippocampus (van den Tweel et al., 2005). The maximal total score for a normal brain was 33.

For Fluoro-Jade B staining, deparaffinized sections were pretreated with $0.06 \%$ potassium permanganate and stained with $0.001 \%$ FluoroJade B-solution (Millipore Bioscience Research Reagents, Hampshire, UK) in $0.1 \%$ acetic acid.

Immunohistochemistry. Deparaffinized sections were incubated with mouse anti-microtubule-associated protein 2 (MAP2) (clone HM-2; Sigma-Aldrich, Steinheim, Germany), mouse anti-myelin basic protein (MBP) (Sternberger Monoclonals, Lutherville, MD), rabbit anti-GRK2 (SC-562; Santa Cruz Biotechnology, Santa Cruz, CA), rat anti-mouseCD68 (Serotec, Oxford, UK), rabbit anti-Ibal (Wako Chemicals, Rich- mond, VA), or rabbit anti-PMN (Accurate Antibodies) antibodies followed by biotinylated horse anti-mouse (Vector Laboratories, Burlingame, CA), mouse anti-rat (Jackson ImmunoResearch, Cambridgeshire, UK), or goat anti-rabbit (Vector Laboratories) antibodies. Visualization was performed using Vectastain ABC kit (Vector Laboratories) and diaminobenzamidine.

Analysis of damage. Full section images at $-1.8 \mathrm{~mm}$ (at hippocampal level) and $+0.7 \mathrm{~mm}$ (at striatal level) from the bregma were captured with a Nikon (Tokyo, Japan) D1 digital camera. The various brain areas were outlined manually using image processing tools in Adobe Photoshop 6.0 (Adobe Systems, San Jose, CA) or NIH ImageJ software, and the ratio of ipsilateral to contralateral areas was calculated (Nijboer et al., 2007).

Preparation of brain homogenates. Pups were decapitated at 3-48 h post-HI, the cerebellum was removed, and the left and right hemispheres were frozen in liquid nitrogen, pulverized using a liquid nitrogen-cooled mortar and pestle, divided into two fractions, and stored at $-80^{\circ} \mathrm{C}$.

Pulverized hemispheres were homogenized in $70 \mathrm{~mm}$ sucrose, 210 mM mannitol, 5 mM HEPES, 1 mM EDTA, and protease inhibitors using a Potter homogenizer (Heidolph, Schwabach, Germany) Mitochondrion-free cytosolic and mitochondrion-enriched fractions were prepared as described previously (Nijboer et al., 2007). Quality of subcellular fractions was verified by immunoblotting with specific markers. Protein concentration was determined using a protein assay (Bio-Rad, Hercules, CA).

Western blotting. Proteins were separated by SDS-PAGE and transferred to nitrocellulose membranes (Hybond-C; GE Healthcare, Roosendaal, The Netherlands). Equal protein loading was verified by Ponceau-S staining.

Membranes were stained with rabbit anti-GRK2 (Santa Cruz Biotechnology), mouse anti-cytochrome $c$ (BD Biosciences, San Jose, CA), rabbit anti-cleaved caspase 3, rabbit anti-Bcl-2, or rabbit anti-Bcl-xL (all from Cell Signaling Technology, Danvers, MA), followed by incubation with donkey $\alpha$-rabbit-HRP (GE Healthcare) or goat anti-mouse-HRP (Jackson ImmunoResearch, Cambridgeshire, UK). To control for equal loading, membranes were stripped and reprobed for $\beta$-actin in cytosolic fraction. Ponceau $S$ staining was used to verify equal loading in mitochondrial fractions, because cytochrome $c$ oxidase complex IV (COX IV) expression was affected by HI.

Specific bands were visualized by chemiluminescence detection (ECL; GE Healthcare) with $\mathrm{x}$-ray film exposure. Films were scanned with a GS-700 Imaging Densitometer and analyzed with Quantity One Software (both from Bio-Rad).

Neutrophil infiltration. Myeloperoxidase (MPO) activity was determined as described previously (Eijkelkamp et al., 2007). In short, pulverized brains were homogenized in $50 \mathrm{mM}$ HEPES buffer, $\mathrm{pH} 8.0$, and centrifuged, and pellets were rehomogenized in $\mathrm{H}_{2} \mathrm{O} / 0.5 \%$ cetyltrimethylammonium chloride (CTAC) (Merck, Darmstadt, Germany). After centrifugation, supernatants were diluted in $10 \mathrm{~mm}$ citrate buffer, $\mathrm{pH}$ 5.0/0.22\% CTAC, and substrate solution [3 $\mathrm{mm} \quad 3^{\prime}, 5,5^{\prime}$ tetramethylbenzidine dihydrocloride (TMB) (Sigma-Aldrich), $120 \mu \mathrm{M}$ resorcinol (Merck), and $2.2 \mathrm{~mm}_{2} \mathrm{O}_{2}$ in distilled water] was added. These reaction mixtures were incubated for $30 \mathrm{~min}$ at $37^{\circ} \mathrm{C}$ and stopped by addition of $\mathrm{H}_{2} \mathrm{SO}_{4}$. Optical density (OD) at $450 \mathrm{~nm}$ was determined. MPO activity determined in samples of a known number of neutrophils was used as reference. Protein concentration of samples was determined with BCA protein assay (Pierce, Rockford, IL) using BSA as standard.

Cerebral blood flow. The reduction in ipsilateral blood flow was measured (Gilland and Hagberg, 1996). A dose of $8 \mu \mathrm{Ci}$ of iodo- $\left[{ }^{14} \mathrm{C}\right]-$ antipyrine $(1600 \mu \mathrm{Ci} / \mathrm{kg})$ in $0.1 \mathrm{ml}$ of saline was injected subcutaneously in the neck at the end of the hypoxic period, and the pups were decapitated $60 \mathrm{~s}$ after injection. The brain was removed in $<35 \mathrm{~s}$ to minimize postmortem diffusion (Jay et al., 1988) and ipsilateral and contralateral hemispheres were frozen in liquid nitrogen. Hemispheres were homogenized, and radioactivity was counted in a liquid scintillation counter. Relative ipsilateral blood was expressed as follows: (cpm ipsilateral/cpm contralateral) $\times 100 \%$.

Hippocampal slices. Organotypic hippocampal slices were prepared using the method of Stoppini et al. (1991) from 7-d-old GRK2 ${ }^{+/-}(n=$ 


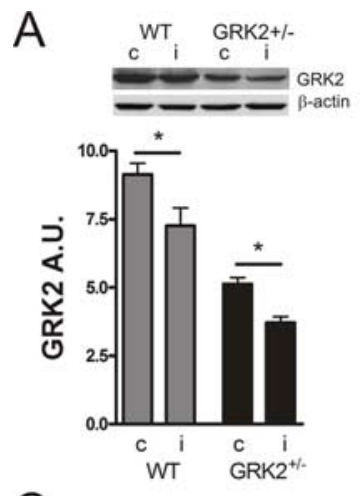

C

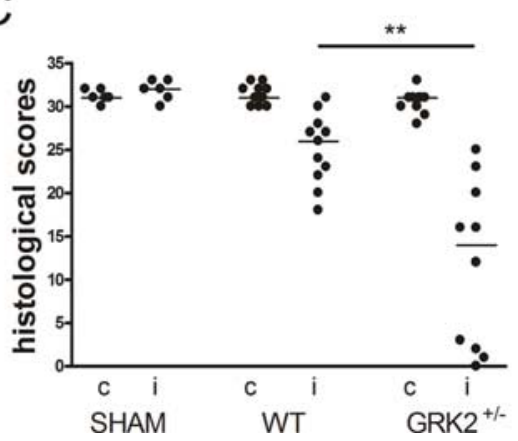

B

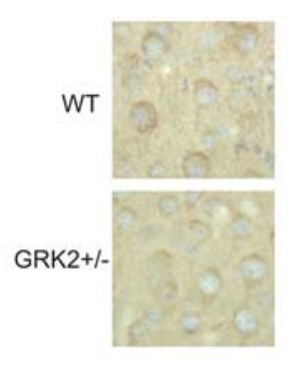

$E$
D

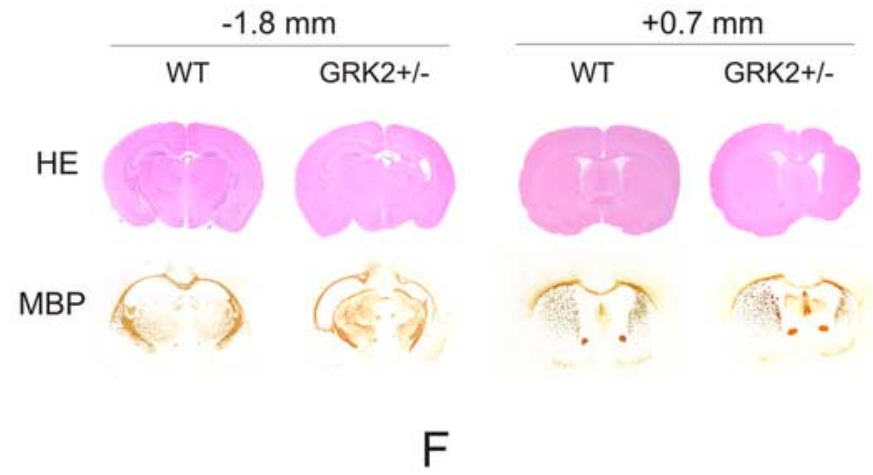

Figure 1. HI-induced brain damage. $A$, GRK2 expression in total brain homogenates was $\sim 50 \%$ lower in GRK2 ${ }^{+/-}$brains compared with WT (contralateral hemispheres WT vs GRK2 ${ }^{+1-}$ ). WT and GRK2 ${ }^{+/-}$mouse pups were exposed to unilateral carotid artery occlusion and 45 min of hypoxia. GRK2 expression level was reduced in ipsilateral hemispheres at $24 \mathrm{~h}$ after $H \mathrm{l}$ in both WT and GRK2 $^{+/-}$animals. ${ }^{*} p<0.05$ versus contralateral levels; $n=6-10$ per group. c, Contralateral; i, ipsilateral; A.U., arbitrary density units. $\boldsymbol{B}$, Photographs illustrating reduced levels of GRK2 in neurons of the parietal cortex in GRK2 ${ }^{+/-}$mice compared with WT animals. C, Brains were collected 3 weeks after the insult. Neuronal integrity was scored in six regions of parietal cortex and five regions of hippocampus on HE-stained coronal sections from WT and GRK2 ${ }^{+/-}$animals. GRK2 ${ }^{+/-}$animals show significant lower scores than WT animals $(* * p<0.01)$. Maximal scores of normal brain tissue: 33 . SHAM, Sham-operated control animals. $\boldsymbol{D}$, Brains were analyzed at two coronal levels: $-1.8 \mathrm{~mm}$ (hippocampal level) or $+0.7 \mathrm{~mm}$ (striatal level) from bregma. MBP, Myelin basic protein immunohistochemistry. $\boldsymbol{E}$, HI induced significantly more hemispheric and hippocampal area loss in the ipsilateral hemisphere of GRK2 ${ }^{+/-}$versus WT mice at 3 weeks post-HI. Hemispheric or hippocampal area loss was determined by comparing areas of ipsilateral to contralateral hemispheres at $-1.8 \mathrm{~mm}$ and $+0.7 \mathrm{~mm}$ from bregma. ${ }^{*} p<0.05{ }^{* *} p<0.01$, WT versus GRK2 ${ }^{+/-}$. $F$, GRK2 ${ }^{+1-}$ mice show significantly more white matter damage than WT littermates at 3 weeks after $\mathrm{Hl}$ at $-1.8 \mathrm{~mm}$ (left) and $+0.7 \mathrm{~mm}$ (right) from bregma. Expression of MBP was determined in contralateral and ipsilateral hemispheres. ${ }^{*} p<0.05 ;{ }^{* *} p<0.01$, WT versus GRK2 ${ }^{+/-}$. Number of animals for $\boldsymbol{C}, \boldsymbol{E}$, and $\boldsymbol{F}$ : WT, $n=11 ;$ GRK2 $^{+/-}, n=10$; and sham controls, $n=6$.

17) and WT $(n=13)$ pups. Using a McIlwain tissue chopper (The Mickle Laboratories, Watford, UK), 400- $\mu$ m-thick hippocampal transverse slices were cut. Slices with complete dentate gyrus and CA3 and CA1 areas were selected and placed (three per membrane) onto $0.4 \mu \mathrm{mol} / \mathrm{L}$ semiporous membranes (Millicell-CM membrane insert; Millipore, Watford, UK). Membranes were placed in Petri dishes of $35 \mathrm{~mm}$ in diameter containing $1 \mathrm{ml}$ of growth medium consisting of $50 \%$ minimal essential medium, $25 \mathrm{mmol} / \mathrm{L}$ HEPES, 25\% heat-inactivated horse serum, $3.5 \%$ sodium bicarbonate, $20 \%$ HBSS, 2 mmol/L L-glutamine, 10 $\mathrm{ml} / \mathrm{L}$ penicillin-streptomycin, and $6 \mathrm{mg} / \mathrm{ml}$ glucose. Cultures were maintained in $95 \%$ air $-5 \% \mathrm{CO}_{2}$ at $37^{\circ} \mathrm{C}$. Medium was changed every $3-4 \mathrm{~d}$, and experiments were performed after $14 \mathrm{~d}$ in vitro (DIV). Slices were treated with $4 \mathrm{~mm}$ glutamate for $24 \mathrm{~h}$ at $37^{\circ} \mathrm{C}$. Cell death was determined using propidium iodine (PI). PI was added to the culture medium $24 \mathrm{~h}$ after addition of glutamate to a final concentration of $5 \mu \mathrm{g} / \mathrm{ml}$. The cultures were incubated for $1 \mathrm{~h}$ and washed three times for $10 \mathrm{~min}$ with PBS. Neuronal damage was then assessed by quantifying the intensity of PI fluorescence using a Leica DM-IRBE inverted fluorescent microscope (Leica, Rijkswijk, The Netherlands). One phase contrast image and one fluorescence image were taken. With an interactive drawing tool, the borders of the slice were outlined on the phase contrast image and then superimposed on the fluorescent PI image. The gray-level intensity of the PI image was assessed using Leica Quantimet software and used as an index of neuronal death. To calculate percentage of neuronal survival, PI intensity in the experimental slices $\left(P_{\text {glut }}\right)$ was corrected for basal PI fluorescence $\left(P_{0}\right.$, before treatment $)$ and maximal cell death in slices treated with $50 \mathrm{~mm}$ glutamate $\left(P_{\max }\right)$, using the following formula: $\left(P_{\text {glut }}\right.$ $\left.-P_{\max }\right) /\left(P_{0}-P_{\max }\right) \times 100$.

Cerebellar granule neurons. Cerebellar granule cells (CGNs) were prepared from 7-d-old WT and GRK2 ${ }^{+/-}$pups. Neurons were seeded on
poly-D-lysine $(15 \mu \mathrm{g} / \mathrm{ml})$-coated 96 -well plates at a density of $0.3 \times 10^{6}$ cells $/ \mathrm{ml}$ and were cultured in MEM supplemented with $30 \mathrm{~mm}$ D-glucose, $25 \mathrm{~mm} \mathrm{KCl}, 10 \% \mathrm{FCS}, 2 \mathrm{mmol} / \mathrm{L}$ L-glutamine, $100 \mathrm{U} / \mathrm{ml}$ penicillin, 100 $\mu \mathrm{g} / \mathrm{ml}$ streptomycin, and $0.25 \mu \mathrm{g} / \mathrm{ml}$ Fungizone. To prevent growth of dividing (glial) cells, fluorodeoxyuridine and uridinecytosine (50 and $150 \mu \mathrm{M}$, respectively) were added to the cultures $24 \mathrm{~h}$ after plating. CGNs were cultured 7 DIV before addition of different concentrations of glutamate for $24 \mathrm{~h}$. MTT $(0.5 \mathrm{mg} / \mathrm{ml})$ (Sigma-Aldrich) was added to the medium for $4 \mathrm{~h}$, MTT crystals were dissolved in DMSO, and OD was measured at $550 \mathrm{~nm}$.

Primary microglia. Primary cultures of cortical microglia were obtained from 1-d-old WT and GRK2 ${ }^{+/-}$mice. Cortices were dissected after removal of meninges and blood vessels, minced and incubated with $0.25 \%$ trypsin for $15 \mathrm{~min}$ in Gey's balanced salt solution containing 100 $\mathrm{U} / \mathrm{ml}$ penicillin, $100 \mu \mathrm{g} / \mathrm{ml}$ streptomycin, and $30 \mathrm{~mm} \mathrm{D}(+)$-glucose. Cells were dissociated and cultured in poly-L-ornithine $(15 \mu \mathrm{g} / \mathrm{ml})$-coated culture flasks in DMEM/Ham's F10 (1:1) supplemented with 10\% FCS, 2 mM glutamine, and antibiotics as stated above. After 10 DIV, flasks were shaken overnight at $37^{\circ} \mathrm{C}$ to detach microglia from the astrocyte layer. Microglia were cultured for $48 \mathrm{~h}$ in poly-L-ornithine-coated 24-well plates at a density of $0.5 \times 10^{6}$ cells $/ \mathrm{ml}$, and total cell lysates were prepared by incubation in ice-cold radioimmunoprecipitation assay buffer (20 mм HEPES, pH 7.5, 1\% Triton X-100, 150 mm NaCl, 10 mм EDTA, and protease inhibitors) for $30 \mathrm{~min}$ at $4^{\circ} \mathrm{C}$ followed by $15 \mathrm{~min}$ centrifugation at 13,000 rpm.

Statistical analysis. Mortality rates were analyzed with Fisher's exact test. Histological scores are presented as individual data with median and were analyzed using Kruskal-Wallis tests. Mann-Whitney $U$ tests were used for additional analysis. All other data are presented as mean and SEM. One-way or two-way ANOVA with Bonferroni's posttests were 
A

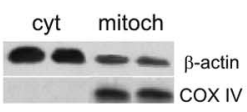

B
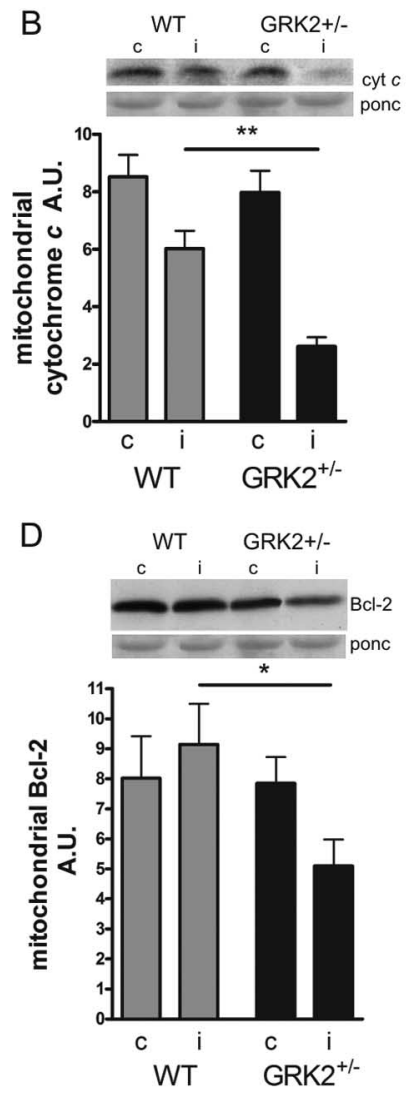

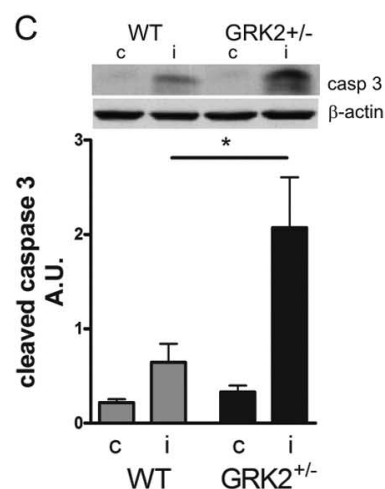

$\mathrm{E}$
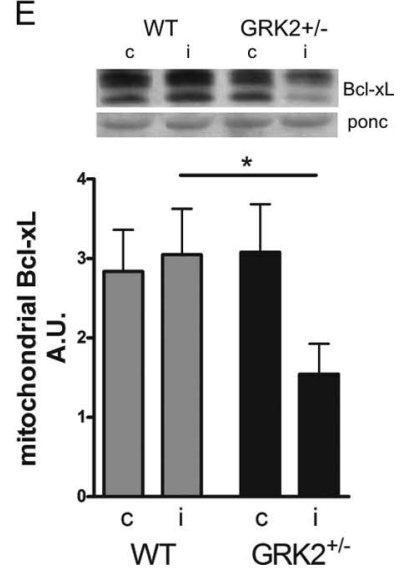

A
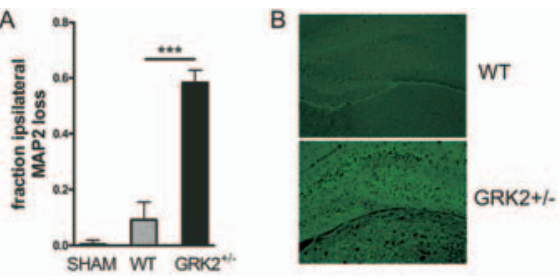

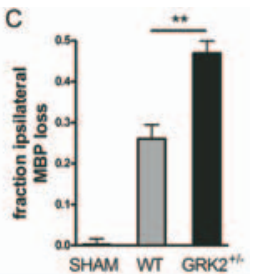

Figure 3. Early neuronal and white matter damage at $24 \mathrm{~h}$ post-HI. A, GRK2 ${ }^{+/-}$animals show significantly more loss of MAP2 staining as an early marker of neuronal damage at $24 \mathrm{~h}$ after HI than WT animals. The ratio of the total area of ipsilateral/contralateral MAP2 was determined at hippocampal level. ${ }^{* * *} p<0.001$. B, Representative example of Fluoro-Jade B staining in hippocampus of WT and GRK2 ${ }^{+/-}$animals at $24 \mathrm{~h}$ after HI. C, GRK2 ${ }^{+/-}$animals show significantly more loss of MBP staining at $24 \mathrm{~h}$ after $\mathrm{HI}$ than WT animals. The ratio of the total area of ipsilateral/contralateral MBP expression was determined at hippocampal level. ${ }^{* *} p<0.01 . A-C, n=10-13$ animals per group; sham, $n=8$.

suggest that there are no major cell-specific differences in the level of reduction in GRK2 expression in GRK2 ${ }^{+/-}$animals.

The data in Figure $1 \mathrm{~A}$ confirm our previous observations in the neonatal rat model that GRK2 is reduced in the ipsilateral hemisphere after HI. Moreover, the relative HI-induced decrease was similar in both genotypes.

\section{Role of GRK2 in brain damage}

Mortality rates during $\mathrm{HI}$ procedures did not differ between genotypes (WT animals, 13.2\%; GRK2 ${ }^{+/-}$animals, 17.0\%; Fisher's exact test, $p=0.46$ )

At 3 weeks post-HI, brain sections were scored for neuronal integrity in six regions of the parietal cortex and in five regions of the hippocampus. The data in Figure $1 C$ demonstrate that there was significantly more damage in ipsilateral parietal cortex and hippocampus $(p<0.01)$ of GRK2 ${ }^{+/-}$animals compared with WT animals. Moreover, GRK2 ${ }^{+/-}$animals displayed more brain damage as determined by measuring areas of ipsilateral and contralateral hippocampus and of the entire hemispheres at -1.8 $\mathrm{mm}$ and $+0.7 \mathrm{~mm}$ from bregma (Fig. $1 D, E$ ). Similar differences in brain damage between WT and GRK2 ${ }^{+/-}$animals were observed after 60 min of hypoxia (data not shown).

To determine the effect of GRK2 on the extent of white matter damage, we determined loss of ipsilateral MBP staining at 3 weeks after the insult. At both $-1.8 \mathrm{~mm}$ and $+0.7 \mathrm{~mm}$ from bregma, loss of MBP staining was more pronounced in $\mathrm{GRK}^{+1-}$ mice compared with WT mice $(p<0.05$ at $-1.8 \mathrm{~mm} ; p<0.01$ at +0.7 $\mathrm{mm})$ (Fig. $1 D, F)$.

\section{Early damage and apoptotic cell death}

To determine whether the observed increase in brain injury in GRK2 $^{+1-}$ animals at 3 weeks post-HI was associated with increased early apoptosis, we prepared subcellular fractions (Fig. $2 \mathrm{~A}$ ) and determined the reduction in mitochondrial cytochrome $c$ levels and the level of activated caspase 3 , an important apoptosis executioner at $24 \mathrm{~h}$ post-HI. GRK2 ${ }^{+/-}$pups showed a significantly larger reduction of ipsilateral mitochondrial cytochrome $c$ levels $(p<0.01)$ and a significantly higher level of ipsilateral activated caspase 3 than WT littermates $(p<0.05)$ (Fig. $2 B, C$ ). In addition, ipsilateral levels of the anti-apoptotic molecules Bcl-2 and Bcl-xL, which are important guardians of the mitochondrial membrane, were significantly reduced after $\mathrm{HI}$ in GRK2 ${ }^{+1-}$ mice but not in WT mice $(p<0.05)$ (Fig. $\left.2 D, E\right)$. We did not detect any cytochrome $c$ leakage, caspase 3 activation, or changes in $\mathrm{Bcl}-2$ and $\mathrm{Bcl}-\mathrm{xL}$ in the contralateral hemisphere of

reductions in GRK2 levels were observed throughout the brain (e.g., in neurons of the hippocampus). Collectively, these data

\section{Results \\ GRK2 expression}

We have shown previously that expression of GRK2 protein was (Kleibet al., 2004) as well as in primary cultures of astroc also reduced by $\sim 50 \%$ in total homogenates of the brain after removal of cerebellum (Fig. $1 A$ ) and by $\sim 42 \%$ in primary cultures of microglia.

The data in Figure $1 B$ illustrate that GRK2 was also reduced in neurons of the parietal cortex from GRK2 ${ }^{+/-}$animals. Similar 
WT and GRK2 ${ }^{+/-}$animals compared with sham-operated animals (Fig. $2 B-E$, insets).

In line with increased signs of apoptosis, we observed more pronounced loss of MAP2 and increased Fluoro-Jade B staining at $24 \mathrm{~h}$ after the insult, indicating that increased damage was already present at this time point (Fig. 3A,B). Moreover, white matter damage, determined by examining the ratio of ipsilateral over contralateral MBP staining at $24 \mathrm{~h}$ after $\mathrm{HI}$, was more pronounced in GRK2 ${ }^{+/-}$animals (Fig. 3C).

\section{Cerebral blood flow}

It is known that GRK2 plays an important role in the regulation of blood flow and arterial blood pressure (Hansen et al., 2006). Therefore, we analyzed whether increased brain damage in GRK2 ${ }^{+/-}$animals was associated with a more pronounced reduction in blood flow in the ipsilateral hemisphere. We did not observe differences in the HI-induced reduction in cerebral blood flow in the ipsilateral hemisphere between WT (54.8 $\pm 7.7 \%$ reduction) and $\mathrm{GRK}^{+/-}(49.3 \pm 8.0 \%$ reduction $)$ animals.

\section{Neutrophil infiltration}

Neutrophils are known to contribute significantly to $\mathrm{HI}$-induced brain damage (Hudome et al., 1997; Palmer et al., 2004). In addition, there is evidence that GRK2 can regulate chemotactic activity (Vroon et al., 2006). Therefore, we first set out to investigate whether the increased damage in GRK $2^{+/-}$animals was caused by increased recruitment of neutrophils to the brain. At 24 and $48 \mathrm{~h}$ after the insult, the HI-induced neutrophil infiltration, determined as cerebral MPO activity, was significantly more pronounced in GRK2 ${ }^{+/-}$mice than in WT littermates (Fig. $\left.4 A\right)(p<0.01 ; p<$ $0.001)$. In addition, only in GRK $2^{+/-}$animals, some MPO activity was already detectable at $6 \mathrm{~h}$ post-HI (Fig. $4 \mathrm{~A}$ ). No MPO activity was detectable in brains of sham controls.

To further delineate the contribution of neutrophils to the increase in brain damage in GRK2 ${ }^{+/-}$animals, we depleted the animals of neutrophils by intraperitoneal administration of a polyclonal anti-neutrophil antibody. This procedure efficiently prevented neutrophil mobilization to the blood and the brain (Fig. 4B-D). Neutrophil depletion significantly attenuated MAP2 loss at $48 \mathrm{~h}$ after the insult in both genotypes, confirming that neutrophilic granulocytes play an important role in the development of HI brain damage (Fig. $4 E)(p<0.05 ; p<0.001)$. Interestingly, however, the differences in MAP2 loss between WT and GRK2 ${ }^{+1-}$ mice at $48 \mathrm{~h}$ after the insult were maintained after neutrophil depletion (Fig. $4 E)(p<0.01)$.

\section{Kinetics of development of gray and white matter damage}

Next, we examined the kinetics of the development of brain damage in WT and GRK2 ${ }^{+/-}$animals in more detail. Interestingly, the first signs of neuronal loss in GRK2 ${ }^{+/-}$animals were already observed before MPO activity could be detected in the brain; already at $3 \mathrm{~h}$ after $\mathrm{HI}$, significant MAP2 loss was detected in $\mathrm{GRK}^{+/-}$animals $(p<0.05)$, whereas in WT animals, we did
B
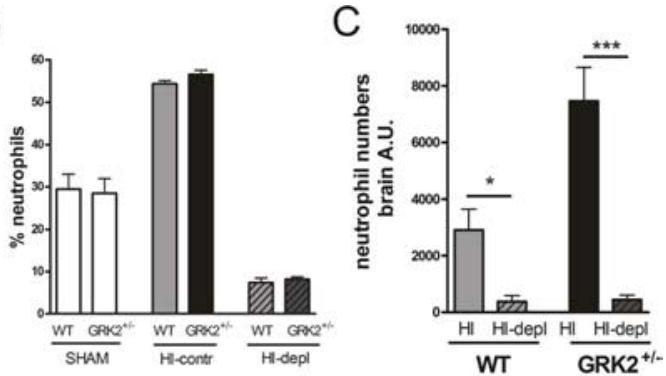

E

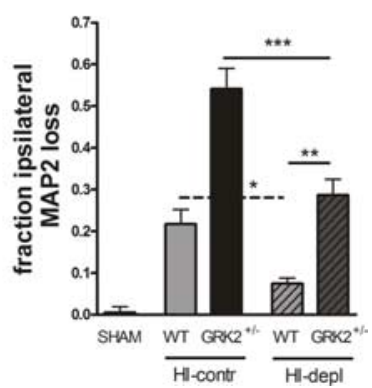

GRK2+/-
WT

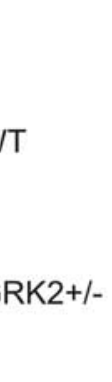

HI-depl

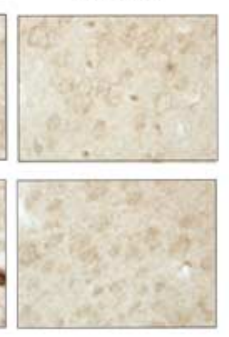
the ipsilateral hemisphere at $6-48 \mathrm{~h}$ post-HI as a measure of neutrophil mobilization to the brain. Brain neutrophil numbers are significantly higher in GRK2 ${ }^{+/-}$mice versus WT mice at $24-48 \mathrm{~h}$ post-HI. ${ }^{* *} p<0.01,{ }^{* * *} p<0.001$. Animal numbers: $6-10$ per group. $\boldsymbol{B}-\boldsymbol{D}, \mathrm{GRK} 2^{+/-}$and WT animals were treated with anti-neutrophil serum (depl) or control serum (contr) at $20 \mathrm{~h}$ pretreatment with anti-neutrophil antibodies on $\mathrm{HI}$-induced MAP2 loss at $48 \mathrm{~h}$ after $\mathrm{HI}$ in GRK2 ${ }^{+-}$and WT animals. In both genotypes, neutrophil depletion (depl) significantly reduced MAP2 Ioss (WT, ${ }^{*} p<0.05$;GRK2 ${ }^{+/-},{ }^{* *} p<0.001$ ). Importantly,

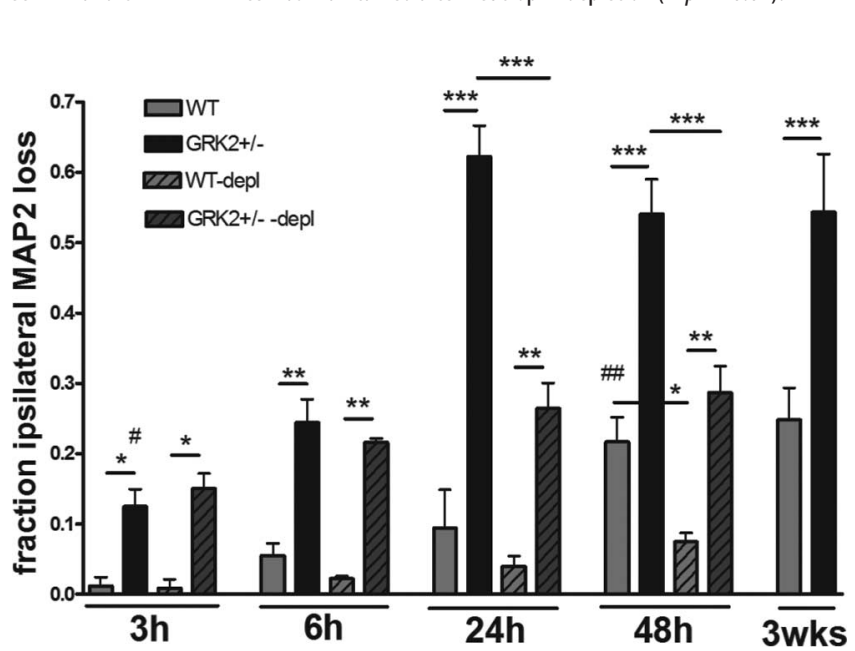

Figure 5. Effect of neutrophil depletion on kinetics of HI-induced MAP2 loss. GRK2 ${ }^{+/-}$and WT animals were treated with anti-neutrophil antiserum (depl) or control serum as described in Figure $4 B-D$. Animals were exposed to $\mathrm{HI}$ and killed at the time points indicated. Total ipsilateral MAP2 loss was determined. Significant MAP2 loss was first detectable at $3 \mathrm{~h}$ in GRK2 $2^{+1-}$ mice $\left({ }^{\#} p<0.05\right)$ and at $48 \mathrm{~h}$ in WT mice $\left({ }^{\# \#} p<0.01\right)$. In both genotypes, MAP2 loss at 48 h was similar to MAP2 loss at 3 weeks after HI. MAP2 loss was not affected by neutrophil depletion at 3-6h post-HI. At 24-48 h post-HI, neutrophil depletion reduced MAP2 loss. ${ }^{*} p<0.05,{ }^{* *} p<$ $0.01,{ }^{* * *} p<0.001$. See also Figure $4 E$. Number of animals: $6-13$ per group.

not detect any MAP2 loss before $48 \mathrm{~h}$ after HI $(p<0.01)$ (Fig. 5$)$. In both genotypes, MAP2 loss at 3 weeks was similar to MAP2 loss at $48 \mathrm{~h}$ after HI.

Moreover, the extent of MAP2 loss in GRK2 ${ }^{+/-}$animals at 3 and $6 \mathrm{~h}$ after HI was not affected by depletion of neutrophils, 
A

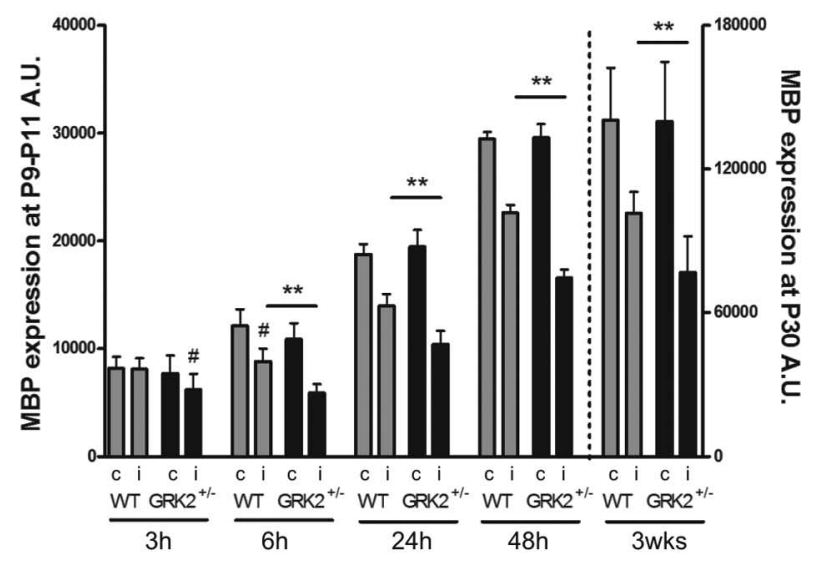

B

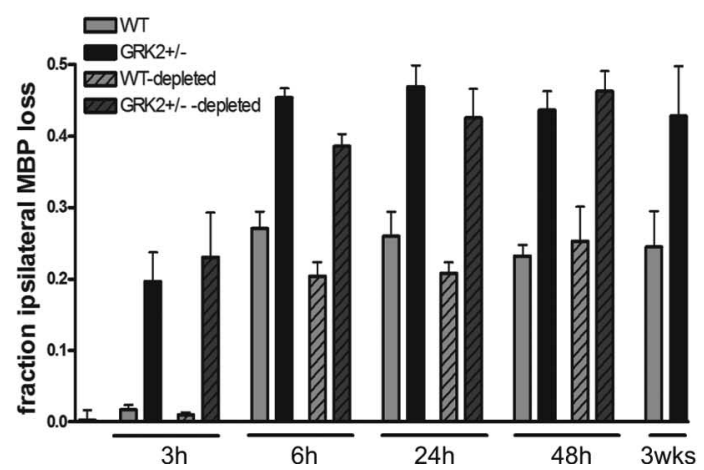

Figure 6. Kinetics of MBP loss after $\mathrm{HI}$ and the effect of neutrophil depletion. $\boldsymbol{A}$, Expression of MBP in contralateral (C) and ipsilateral (i) hemispheres at different time points after HI: $3-48 \mathrm{~h}$ (left $y$-axis) and 3 weeks (right $y$-axis). Significant loss of ipsilateral MBP expression was first detectable at $3 \mathrm{~h}$ in GRK2 ${ }^{+/-}$mice and at $6 \mathrm{~h}$ in WT mice ( $\left.{ }^{\#} p<0.05\right)$. GRK2 ${ }^{+/-}$mice showed significantly more loss of ipsilateral MBP expression than WT mice at all time points $\left({ }^{* *} p<\right.$ $0.01 ; n=7-11$ animals per group). $\boldsymbol{B}$, The relative reduction of ipsilateral MBP expression did not increase from $6 \mathrm{~h}$ to 3 weeks post-HI in both genotypes [solid gray (WT) and black $\left(G R K 2^{+/-}\right.$) bars]. Neutrophil depletion (see Fig. $4 B-D$ ) did not affect loss of MBP expression in the ipsilateral hemisphere [hatched light gray (WT) and hatched dark gray (GRK2 ${ }^{+/-}$) bars].

indicating that at this early time point, neuronal damage developed independently of neutrophil infiltration (Fig. 5).

In both GRK2 ${ }^{+/-}$and WT animals, myelination of white matter tracts was clearly less developed at $3 \mathrm{~h}$ after the insult than at later time points $(24-48 \mathrm{~h}$ to 3 weeks after HI) (Fig. $6 \mathrm{~A}$ ). Contralateral MBP staining in this time frame did not differ between genotypes. In GRK $2^{+/-}$mice, however, a $\sim 20 \%$ reduction of ipsilateral/contralateral MBP staining could already be observed at 3 h (Fig. $6 A)(p<0.05)$. Significant MBP loss was first detectable in WT animals at $6 \mathrm{~h}$ post-HI (Fig. 6A) $(p<0.05)$. GRK2 ${ }^{+/-}$mice showed significantly more MBP loss $(\sim 45 \%)$ than WT littermates $(\sim 25 \%)$ at $6-48 \mathrm{~h}$ post-HI $(p<0.01)$ (Fig. $6 A, B)$. Interestingly, however, the relative reduction in ipsilateral MBP staining did not increase between $6 \mathrm{~h}$ and 3 weeks after $\mathrm{HI}$ in both genotypes. These data indicate that the maximal relative reduction in MBP was reached already very early $(6 \mathrm{~h})$ after $\mathrm{HI}$ in both genotypes (Fig. 6B) (nondepleted bars).

We discovered that, in contrast to what had been observed for neuronal damage, depletion of neutrophils did not affect loss of $\mathrm{MBP}$ expression at 3-48 h post-HI. The latter suggests that the $\mathrm{HI}$-induced increase in cerebral neutrophils does not contribute to early white matter damage (Fig. 6B) (depleted bars).

\section{Activated microglia/monocytes}

To investigate whether increased and more advanced activation and migration of microglia/macrophages could contribute to earlier and more pronounced neuronal loss in animals deficient for GRK2, we analyzed CD68 staining. In both WT and GRK2 ${ }^{+/-}$ animals, we could not detect any HI-induced changes in CD68 expression at $6 \mathrm{~h}$ after HI. At 24 and $48 \mathrm{~h}$ after HI, the HI-induced upregulation of CD68 expression was more pronounced in GRK2 ${ }^{+/-}$animals than in WT animals (Fig. $7 A$ ). The relative difference in microglia activation between GRK2 ${ }^{+/-}$animals and WT animals was similar in the hippocampus and cortex (data not shown). These data suggest that increased microglial activation, as observed in GRK2 ${ }^{+-}$animals, occurs as a reactive process induced by increased brain damage. Therefore, increased microglial activation is not the primary cause of increased damage in GRK2 ${ }^{+/-}$animals. As shown in Figure $7 B$, neutrophil depletion did not have major effects on HI-induced activation of microglia.

\section{In vitro sensitivity to glutamate-induced cell death}

Dale et al. (2000) have shown that overexpression of GRK2 can protect against mGluR1-mediated cell death in human embryonic kidney 293 cells. In this study, we analyzed whether a reduction in endogenous GRK2 has consequences for glutamateinduced cytotoxicity in ex vivo cultures of hippocampal slices. Indeed, we observed a small, but statistically significant ( $p=$ 0.02 ) $22 \%$ decrease in cellular survival after exposure to glutamate in GRK2 ${ }^{+/-}(45.1 \pm 4.3 \%$ survival $)$ compared with WT ( $57.9 \pm 2.8 \%$ survival) hippocampal slices. Finally, we also compared the sensitivity of primary cultures of CGN from WT and GRK2 ${ }^{+/-}$animals to glutamate-induced cell death. Glutamate dose-dependently induced cell death (Fig. 8). Moreover, the data in Figure 8 clearly demonstrate that glutamate-induced cell death was significantly more pronounced in cultures of GRK2 ${ }^{+/-}$CGN compared with WT CGN.

\section{Discussion}

GPCR comprise the largest known family of cell surface receptors. Malfunction or dysregulation of GPCR is a major contributor to the pathophysiology of disease and $>50 \%$ of all therapeutics target GPCR-mediated pathways (Flower, 1999). Here, we present the novel concept that GRK2, a ubiquitous member of the GRK family that plays a central role in GPCR regulation, represents an important endogenous factor in the protection against HI brain damage. We demonstrate that the $50 \%$ reduction in GRK2 protein expression in GRK2 ${ }^{+/-}$mouse pups markedly exacerbated long-term gray and white matter damage after HI. Moreover, we show that partial GRK2 deficiency accelerated onset of early neuronal and white matter damage after a $\mathrm{HI}$ insult in neonatal mice.

Systemic neutrophil depletion did clearly reduce neuronal loss in both genotypes, confirming previous observations that neutrophils are important contributors to the extent of neuronal loss in this model (Hudome et al., 1997; Palmer et al., 2004). Because GRK2 is involved in chemotaxis (Vroon et al., 2006), we investigated the contribution of neutrophil infiltration to the increase in gray and white matter damage in GRK2 ${ }^{+/-}$animals. Importantly, neutrophil depletion did not prevent the early onset of neuronal damage we observed in GRK2 ${ }^{+/-}$animals at $3-6 \mathrm{~h}$ after HI. Moreover, the increased level of damage was maintained in neutrophil-depleted GRK2 ${ }^{+/-}$animals, although neutrophil depletion did prevent the large further increase in damage that was observed between 6 and 48 h post-HI. In intact GRK2 ${ }^{+/-}$ animals, the first signs of neutrophil infiltration in the brain were 


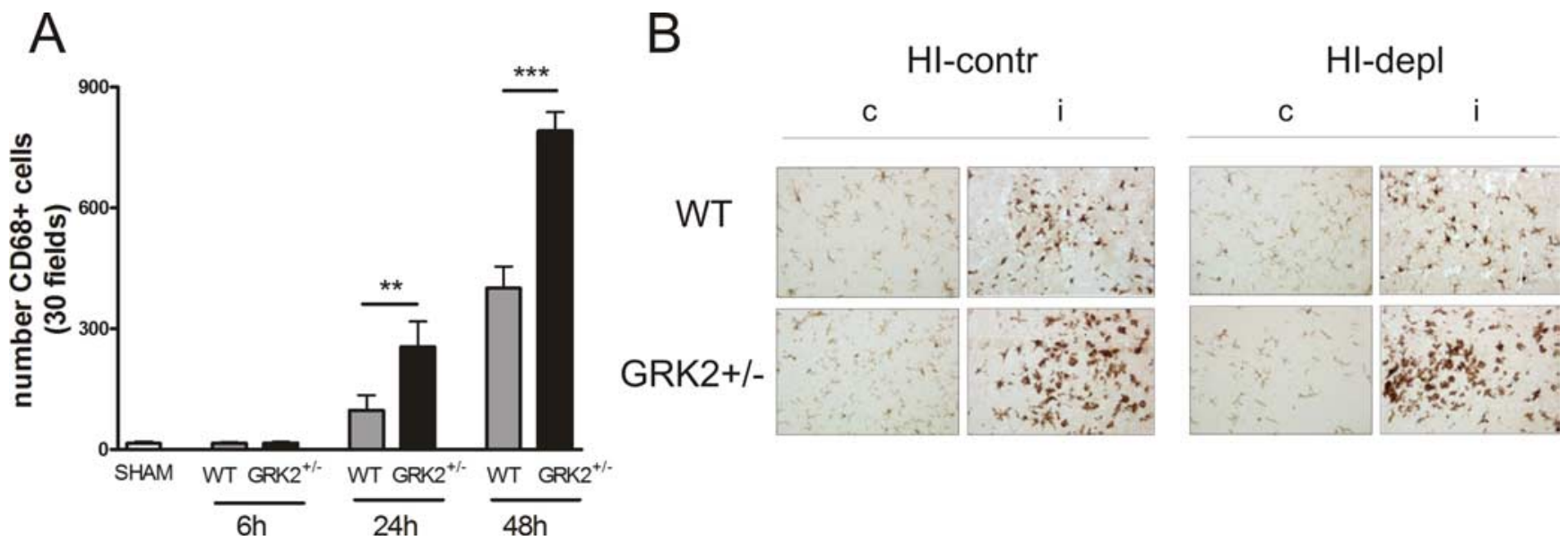

Figure 7. HI-induced increase in CD68 expression. A, CD68-positive cells were counted in six visual fields of hippocampus and 24 visual fields of parietal cortex. At $24-48 \mathrm{~h} \mathrm{post-HI,} \mathrm{GKR2}{ }^{+/-}$ mice show significantly higher numbers of CD68-positive cells in the brain than WT littermates ( ${ }^{* *} p<0.01$; ${ }^{* *} p<0.001$ ). Number of animals: 7 per group. $\boldsymbol{B}$, Representative examples showing that neutrophil depletion did not affect microglial activation in WT and GRK2 ${ }^{+/}$animals. Slices obtained at $48 \mathrm{~h}$ post-HI were stained for Iba-1. depl, Treated with anti-neutrophil serum; contr, control serum.

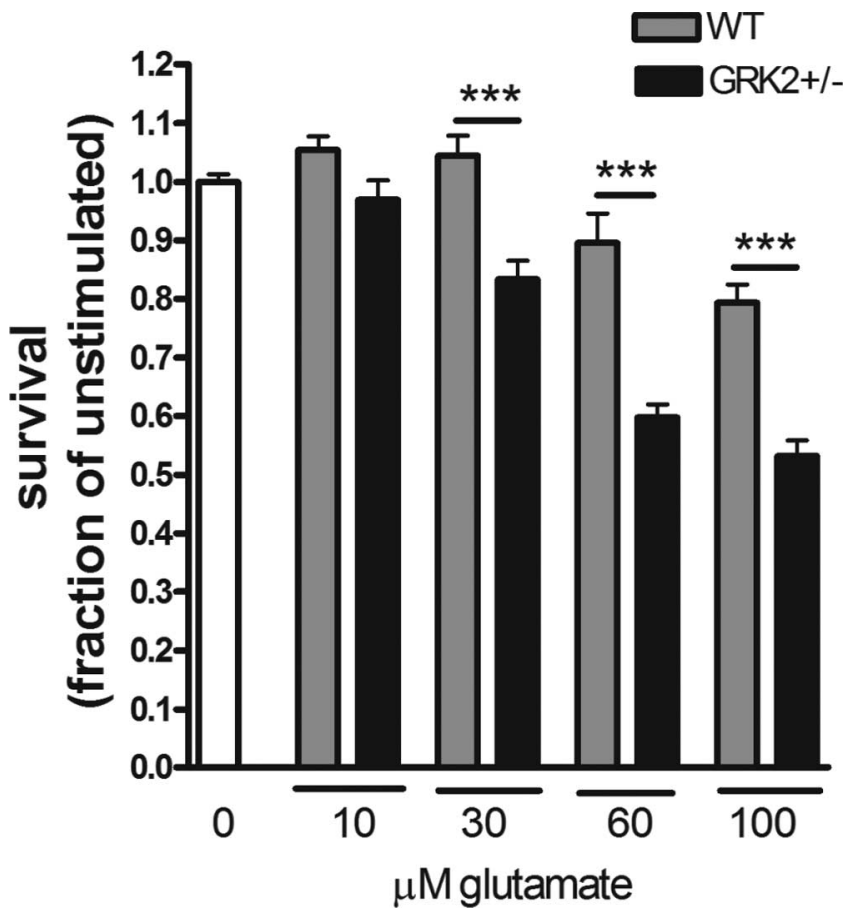

Figure 8. Increased sensitivity to glutamate in vitro. Primary CGNs were cultured for $7 \mathrm{DIV}$ before inducing cell death by different concentration of glutamate for $24 \mathrm{~h}$. Cell death was determined using MTT assay. GRK2 ${ }^{+1-}$ CGNs are significantly more vulnerable to glutamate than WT CGNs (*** $p<0.001)$. Number of animals: WT, $n=6$; GRK2 ${ }^{+/-}, n=5$. All experiments were performed in sixfold.

detectable at $6 \mathrm{~h}$ after $\mathrm{HI}$ and were associated with a sharp increase in the amount of MAP2 loss between 6 and $24 \mathrm{~h}$ after the insult. Collectively, our data suggest that the increased distribution of neutrophils to the brain of GRK2 ${ }^{+/-}$animals is in fact the result of a reactive process and secondary to neuronal damage. These neutrophils exacerbate the already existing neuronal damage but are not the primary cause of the more pronounced neuronal loss in GRK2 ${ }^{+/-}$mice.

We show here for the first time that neutrophil depletion did not change the level of MBP staining during the observation period of 3-48 h after $\mathrm{HI}$, suggesting that neutrophils do not con- tribute to HI-induced early white matter loss. In line with this observation, the more pronounced HI-induced decrease in ipsilateral MBP in GRK2 ${ }^{+/-}$versus WT animals appeared to be independent of neutrophils as well.

Our present data confirm previous suggestions that cerebral microglial/macrophage activation after $\mathrm{HI}$ is part of a reactive process, because appearance of activated microglia in the brain clearly followed the onset of neuronal loss (McRae et al., 1995). Therefore, we propose that microglia/macrophage infiltration and activation is not the primary cause of increased and advanced early damage in the GRK2 ${ }^{+/-}$animals but may well contribute to further aggravation of brain damage in these animals.

Based on the depletion studies and analysis of the kinetics of development of brain damage and of cerebral blood flow, we propose that the earlier and more pronounced cerebral damage in GRK2 ${ }^{+/-}$animals results from increased sensitivity of gray and white matter to factors produced in response to the insult. However, the mechanisms leading to increased damage caused by low GRK2 levels will include multiple pathways that may be different for gray and white matter. GRK2 regulates the activity of multiple GPCR in the brain, including mGluR1 and mGluR5 (Dale et al., 2000; Dhami et al., 2002; Pellegrini-Giampietro, 2003; Sorensen and Conn, 2003) and chemokine receptors. Excessive glutamate release and glutamate excitotoxicity are thought to be key to the initiation of the process leading to neuronal loss after cerebral HI. In addition, immature oligodendroglial cells are highly susceptible to glutamate excitotoxicity (Follett et al., 2000). Interestingly, it has been shown that overexpression of GRK2 can protect cells in vitro against mGluR1dependent cell death and reduces glutamate-induced signaling via both mGluR1 and mGluR5. Both neurons and oligodendroglial cells express metabotropic glutamate receptors, and there is also evidence that mGluR1 antagonists can reduce NMDA receptor-mediated neuronal death (Strasser et al., 1998). Therefore, it may well be possible that reduced levels of GRK2 facilitate excitotoxic damage by overstimulation of mGluRs, either directly or indirectly via potentiation of NMDA receptor signaling. Our ex vivo studies using hippocampal slices or CGN from WT and $\mathrm{GRK}^{+/-}$animals indeed showed that partial deletion of GRK2 significantly increased the sensitivity for glutamate-induced cell death. However, it should be noted that in vivo, not only gluta- 
mate receptors but also other GRK2 substrate receptors may contribute to the observed difference in neuronal loss after HI.

Apart from its classical role in receptor desensitization, more recent evidence suggests that GRK2 can also interfere with cellular signaling and activity at a more downstream level. For example, GRK2 can interact directly with MAP kinase kinase (MEK), p38, and Akt, and these interactions inhibit phosphorylation of downstream substrates of these kinases (Naga Prasad et al., 2001; Liu et al., 2005; Jiménez-Sainz et al., 2006; Peregrin et al., 2006; Kleibeuker et al., 2008). Moreover, there is evidence that the phosphatidyl inositol 3-kinase-Akt pathway as well as the MEKERK (extracellular signal-regulated kinase) pathway is important for the regulation of neuronal apoptosis and survival, indicating that direct interactions of GRK2 with these molecules may affect cell survival (Frebel and Wiese, 2006). GRK2 also binds to cytoskeletal proteins, including tubulin, synuclein and ezrin, and it has been suggested that GRK2 may contribute to the regulation of cytoskeletal reorganization in response to GPCR signaling (Pitcher et al., 1998b; Cant and Pitcher, 2005). In addition, we have shown recently that treatment of human $\mathrm{T}$ cells with the microtubule stabilizer taxol rescues $\mathrm{GRK} 2^{+/-}$cells from deficient agonist-induced internalization of the $\beta$-adrenergic receptor (Vroon et al., 2007). Interestingly, there is evidence that microtubule dissociation precedes MAP2 loss and that the microtubule stabilizer taxol can reduce neuronal damage in a hippocampal slice model of excitotoxic neuronal injury (Hoskison and Shuttleworth, 2006). The consequences of reduced GRK2 for cytoskeletal changes and neuronal damage remain to be determined, but it is conceivable that the earlier loss of the neuronal microtubule-associated protein MAP2 in animals with low GRK2 is caused by direct interactions of GRK2 with cytoskeletal elements.

In a previous study, we described that exposure of neonatal rats to $\mathrm{HI}$ induces a decrease in cerebral GRK2 expression that could be detected prior to the onset of neuronal MAP2 loss in this model. Here, we confirm HI-induced reduction in GRK2 and demonstrate that decreased GRK2 expression sensitizes the brain for HI-induced neuronal and white matter loss. Collectively, these data suggest that alterations in the expression of GRK2 do occur as part of the pathological process after cerebral HI. Interestingly, it has been described in humans that cerebral GRK2 levels decline with age, which might reflect an additional risk factor for brain injury after stroke (Grange-Midroit et al., 2002). Moreover, cerebral GRK2 is relatively low in neonatal rats and increases to adult levels at 3 weeks after birth (Penela et al., 2000).

In conclusion, we show that endogenous GRK2 protects against HI brain damage, because reduced levels of GRK2 accelerate onset and aggravate the extent of HI-induced brain pathology. The underlying mechanisms will be an important subject of our future research. We speculate that prevention of the HIinduced decrease in GRK2 may help to protect the brain against ischemic injury.

\section{References}

Arraes SM, Freitas MS, da Silva SV, de Paula Neto HA, ves-Filho JC, Auxiliadora MM, Basile-Filho A, Tavares-Murta BM, Barja-Fidalgo C, Cunha FQ (2006) Impaired neutrophil chemotaxis in sepsis associates with GRK expression and inhibition of actin assembly and tyrosine phosphorylation. Blood 108:2906-2913.

Buonocore G, Groenendaal F (2007) Anti-oxidant strategies. Semin Fetal Neonatal Med 12:287-295.

Cant SH, Pitcher JA (2005) G protein-coupled receptor kinase 2-mediated phosphorylation of ezrin is required for G protein-coupled receptor- dependent reorganization of the actin cytoskeleton. Mol Biol Cell 16:3088-3099.

Clarkson AN, Sutherland BA, Appleton I (2005) The biology and pathology of hypoxia-ischemia: an update. Arch Immunol Ther Exp (Warsz) 53:213-225.

Cobelens PM, Kavelaars A, Heijnen CJ, Ribas C, Mayor Jr F, Penela P (2007) Hydrogen peroxide impairs GRK2 translation via a calpain-dependent and cdk1-mediated pathway. Cell Signal 19:269-277.

Cowan F, Rutherford M, Groenendaal F, Eken P, Mercuri E, Bydder GM, Meiners LC, Dubowitz LM, de Vries LS (2003) Origin and timing of brain lesions in term infants with neonatal encephalopathy. Lancet 361:736-742.

Dale LB, Bhattacharya M, Anborgh PH, Murdoch B, Bhatia M, Nakanishi S, Ferguson SS (2000) G protein-coupled receptor kinase-mediated desensitization of metabotropic glutamate receptor $1 \mathrm{~A}$ protects against cell death. J Biol Chem 275:38213-38220.

Dhami GK, Anborgh PH, Dale LB, Sterne-Marr R, Ferguson SS (2002) Phosphorylation-independent regulation of metabotropic glutamate receptor signaling by $\mathrm{G}$ protein-coupled receptor kinase 2. J Biol Chem 277:25266-25272.

Eijkelkamp N, Heijnen CJ, Lucas A, Premont RT, Elsenbruch S, Schedlowski M, Kavelaars A (2007) G protein-coupled receptor kinase 6 controls chronicity and severity of dextran sodium sulphate-induced colitis in mice. Gut 56:847-854.

Fan J, Malik AB (2003) Toll-like receptor-4 (TLR4) signaling augments chemokine-induced neutrophil migration by modulating cell surface expression of chemokine receptors. Nat Med 9:315-321.

Ferriero DM (2004) Neonatal brain injury. N Engl J Med 351:1985-1995.

Flower DR (1999) Modelling G-protein-coupled receptors for drug design. Biochim Biophys Acta 1422:207-234.

Follett PL, Rosenberg PA, Volpe JJ, Jensen FE (2000) NBQX attenuates excitotoxic injury in developing white matter. J Neurosci 20:9235-9241.

Frebel K, Wiese S (2006) Signalling molecules essential for neuronal survival and differentiation. Biochem Soc Trans 34:1287-1290.

Gilland E, Hagberg H (1996) NMDA Receptor-dependent increase of cerebral glucose utilization after hypoxia-ischemia in the immature rat. J Cereb Blood Flow Metab 16:1005-1013.

Grange-Midroit M, Garcia-Sevilla JA, Ferrer-Alcon M, La HR, Walzer C, Guimon J (2002) G protein-coupled receptor kinases, beta-arrestin-2 and associated regulatory proteins in the human brain: postmortem changes, effect of age and subcellular distribution. Brain Res Mol Brain Res 101:39-51.

Hansen JL, Theilade J, Aplin M, Sheikh SP (2006) Role of G-proteincoupled receptor kinase 2 in the heart-do regulatory mechanisms open novel therapeutic perspectives? Trends Cardiovasc Med 16:169-177.

Hoskison MM, Shuttleworth CW (2006) Microtubule disruption, not calpain-dependent loss of MAP2, contributes to enduring NMDAinduced dendritic dysfunction in acute hippocampal slices. Exp Neurol 202:302-312.

Hudome S, Palmer C, Roberts RL, Mauger D, Housman C, Towfighi J (1997) The role of neutrophils in the production of hypoxic-ischemic brain injury in the neonatal rat. Pediatr Res 41:607-616.

Jaber M, Koch WJ, Rockman H, Smith B, Bond RA, Sulik KK, Ross Jr J, Lefkowitz RJ, Caron MG, Giros B (1996) Essential role of betaadrenergic receptor kinase 1 in cardiac development and function. Proc Natl Acad Sci USA 93:12974-12979.

Jay TM, Lucignani G, Crane AM, Jehle J, Sokoloff L (1988) Measurement of local cerebral blood flow with [14C] iodoantipyrine in the mouse. J Cereb Blood Flow Metab 8:121-129.

Jiménez-Sainz MC, Murga C, Kavelaars A, Jurado-Pueyo M, Krakstad BF, Heijnen CJ, Mayor Jr F, Aragay AM (2006) G protein-coupled receptor kinase 2 negatively regulates chemokine signaling at a level downstream from $\mathrm{G}$ protein subunits. Mol Biol Cell 17:25-31.

Johnston MV (2005) Excitotoxicity in perinatal brain injury. Brain Pathol $15: 234-240$.

Kleibeuker W, Jurado-Pueyo M, Murga C, Eijkelkamp N, Mayor Jr F, Heijnen CJ, Kavelaars A (2008) Physiological changes in GRK2 regulate CCL2induced signaling to ERK1/2 and Akt but not to MEK1/2 and calcium. J Neurochem 104:979-992.

Liu S, Premont RT, Kontos CD, Zhu S, Rockey DC (2005) A crucial role for GRK2 in regulation of endothelial cell nitric oxide synthase function in portal hypertension. Nat Med 11:952-958. 
Lombardi MS, Kavelaars A, Schedlowski M, Bijlsma JW, Okihara KL, Van de Pol M, Ochsmann S, Pawlak C, Schmidt RE, Heijnen CJ (1999) Decreased expression and activity of G-protein-coupled receptor kinases in peripheral blood mononuclear cells of patients with rheumatoid arthritis. FASEB J 13:715-725.

Lombardi MS, Kavelaars A, Penela P, Scholtens EJ, Roccio M, Schmidt RE, Schedlowski M, Mayor Jr F, Heijnen CJ (2002) Oxidative stress decreases $\mathrm{G}$ protein-coupled receptor kinase 2 in lymphocytes via a calpaindependent mechanism. Mol Pharmacol 62:379-388.

Lombardi MS, van den Tweel E, Kavelaars A, Groenendaal F, van Bel F, Heijnen CJ (2004) Hypoxia/ischemia modulates G protein-coupled receptor kinase 2 and beta-arrestin-1 levels in the neonatal rat brain. Stroke 35:981-986.

Lombardi MS, Vroon A, Sodaar P, van Muiswinkel FL, Heijnen CJ, Kavelaars A (2007) Down-regulation of GRK2 after oxygen and glucose deprivation in rat hippocampal slices: role of the PI3-kinase pathway. J Neurochem 102:731-740.

McRae A, Gilland E, Bona E, Hagberg H (1995) Microglia activation after neonatal hypoxic-ischemia. Brain Res Dev Brain Res 84:245-252.

Naga Prasad SV, Barak LS, Rapacciuolo A, Caron MG, Rockman HA (2001) Agonist-dependent recruitment of phosphoinositide 3-kinase to the membrane by beta-adrenergic receptor kinase 1 . A role in receptor sequestration. J Biol Chem 276:18953-18959.

Nijboer CH, Groenendaal F, Kavelaars A, Hagberg HH, van Bel F, Heijnen CJ (2007) Gender-specific neuroprotection by 2 -iminobiotin after hypoxiaischemia in the neonatal rat via a nitric oxide independent pathway. J Cereb Blood Flow Metab 27:282-292.

Palmer C, Roberts RL, Young PI (2004) Timing of neutrophil depletion influences long-term neuroprotection in neonatal rat hypoxic-ischemic brain injury. Pediatr Res 55:549-556.

Pellegrini-Giampietro DE (2003) The distinct role of mGlul receptors in post-ischemic neuronal death. Trends Pharmacol Sci 24:461-470.

Penela P, Álvarez-Dolado M, Muñoz M, Mayor Jr F (2000) Expression patterns of the regulatory proteins $\mathrm{G}$ protein-coupled receptor kinase 2 and $\beta$-arrestin 1 during rat postnatal brain development: effect of hypothyroidism. Eur J Biochem 267:4390-4396.

Peregrin S, Jurado-Pueyo M, Campos PM, Sanz-Moreno V, Ruiz-Gomez A, Crespo P, Mayor Jr F, Murga C (2006) Phosphorylation of p38 by GRK2 at the docking groove unveils a novel mechanism for inactivating p38MAPK. Curr Biol 16:2042-2047.
Pitcher JA, Freedman NJ, Lefkowitz RJ (1998a) G protein-coupled receptor kinases. Annu Rev Biochem 67:653-692.

Pitcher JA, Hall RA, Daaka Y, Zhang J, Ferguson SS, Hester S, Miller S, Caron MG, Lefkowitz RJ, Barak LS (1998b) The G protein-coupled receptor kinase 2 is a microtubule-associated protein kinase that phosphorylates tubulin. J Biol Chem 273:12316-12324.

Ramos-Ruiz R, Penela P, Penn RB, Mayor Jr F (2000) Analysis of the human G protein-coupled receptor kinase 2 (GRK2) gene promoter: regulation by signal transduction systems in aortic smooth muscle cells. Circulation 101:2083-2089.

Ribas C, Penela P, Murga C, Salcedo A, Garcia-Hoz C, Jurado-Pueyo M, Aymerich I, Mayor Jr F (2007) The G protein-coupled receptor kinase (GRK) interactome: role of GRKs in GPCR regulation and signaling. Biochim Biophys Acta 1768:913-922.

Sorensen SD, Conn PJ (2003) G protein-coupled receptor kinases regulate metabotropic glutamate receptor 5 function and expression. Neuropharmacology 44:699-706

Stoppini L, Buchs PA, Muller D (1991) A simple method for organotypic cultures of nervous tissue. J Neurosci Methods 37:173-182.

Strasser U, Lobner D, Behrens MM, Canzoniero LM, Choi DW (1998) Antagonists for group I mGluRs attenuate excitotoxic neuronal death in cortical cultures. Eur J Neurosci 10:2848-2855.

van den Tweel ER, van Bel F, Kavelaars A, Peeters-Scholte CM, Haumann J, Nijboer CH, Heijnen CJ, Groenendaal F (2005) Long-term neuroprotection with 2-iminobiotin, an inhibitor of neuronal and inducible nitric oxide synthase, after cerebral hypoxia-ischemia in neonatal rats. J Cereb Blood Flow Metab 25:67-74

Vroon A, Heijnen CJ, Raatgever R, Touw IP, Ploemacher RE, Premont RT, Kavelaars A (2004) GRK6 deficiency is associated with enhanced CXCR4-mediated neutrophil chemotaxis in vitro and impaired responsiveness to G-CSF in vivo. J Leukoc Biol 75:698-704.

Vroon A, Kavelaars A, Limmroth V, Lombardi MS, Goebel MU, Van Dam AM, Caron MG, Schedlowski M, Heijnen CJ (2005) G protein-coupled receptor kinase 2 in multiple sclerosis and experimental autoimmune encephalomyelitis. J Immunol 174:4400-4406.

Vroon A, Heijnen CJ, Kavelaars A (2006) GRKs and arrestins: regulators of migration and inflammation. J Leukoc Biol 80:1214-1221.

Vroon A, Lombardi MS, Kavelaars A, Heijnen CJ (2007) Taxol normalizes the impaired agonist-induced beta2-adrenoceptor internalization in splenocytes from GRK2+/- mice. Eur J Pharmacol 560:9-16. 